\title{
Antimicrobial Usage in Smallholder Poultry Production in Nigeria
}

\author{
Oladeji Bamidele $\mathbb{C}^{1,2}$ Tunde A. Amole $\mathbb{D}^{1},{ }^{1}$ Oluwafikayo A. Oyewale $\mathbb{D}^{3}{ }^{3}$ \\ Olayinka O. Bamidele $\mathbb{D D}^{4,5}$ Abdulmojeed Yakubu ${ }^{4},{ }^{6}$ Uduak E. Ogundu ${ }^{\mathbb{D}}{ }^{7}$ \\ Folasade O. Ajayi $\left(\mathbb{D},{ }^{8}\right.$ and Waheed A. Hassan $\mathbb{1}^{9}$ \\ ${ }^{1}$ International Livestock Research Institute (ILRI), P.M.B. 5320, Ibadan 200001, Nigeria \\ ${ }^{2}$ Department of Biological Sciences, Kings University, Odeomu 220104, Nigeria \\ ${ }^{3}$ African Centre of Excellence for Genomics of Infectious Diseases, Redeemer's University, Ede, Nigeria \\ ${ }^{4}$ Faculty of Medicine, Hebrew University, Jerusalem, Israel \\ ${ }^{5}$ Department of Community Medicine, UNIOSUN Teaching Hospital, Osogbo, Osun State, Nigeria \\ ${ }^{6}$ Department of Animal Science, Faculty of Agriculture, Nasarawa State University, Keffi, Shabu-Lafia Campus Lafia, \\ Nasarawa State, Nigeria \\ ${ }^{7}$ Department of Animal Science and Technology, Federal University of Technology, Owerri, Imo State, Nigeria \\ ${ }^{8}$ Department of Animal Science, University of Port-Harcourt, Choba, Rivers State, Nigeria \\ ${ }^{9}$ Department of Animal Science, Usmanu Danfodiyo University, Sokoto, Sokoto State, Nigeria
}

Correspondence should be addressed to Oladeji Bamidele; bamideledeji@gmail.com

Received 25 November 2021; Accepted 12 February 2022; Published 28 February 2022

Academic Editor: Nora Mestorino

Copyright (c) 2022 Oladeji Bamidele et al. This is an open access article distributed under the Creative Commons Attribution License, which permits unrestricted use, distribution, and reproduction in any medium, provided the original work is properly cited.

\begin{abstract}
The indiscriminate use of antimicrobials in livestock production is of increasing concern due to the threat of antimicrobial resistance in both humans and animals. Much emphasis has been placed on intensively managed poultry production systems, which routinely use antimicrobials as against smallholder poultry production systems (SPPS). Therefore, this study investigated the use of antimicrobials among smallholder poultry farmers in Nigeria, and compared the prevalence of antimicrobial drug use against the practice of ethnoveterinary medicine (EVM). A cross-sectional study was conducted in five states (agroecologies) of Nigeria using structured questionnaires administered on a total of 350 farmers. The practice of EVM was prevalent among most of the farmers (39\%). The western method (pharmaceuticals) was practiced by a large proportion of farmers (60\%), either solely (25\%) or in combination with EVM (35\%). Antimicrobials were used primarily for treatment and prevention of diseases (78\%). Semi-scavenging system of production had the highest proportion $(49 \%)$ of farmers using antimicrobials, compared to semiintensive (37\%) and scavenging (14\%) systems. Gender $\left(\chi^{2}=9.30, p=0.01\right)$, and location $\left(\chi^{2}=216.86, p \leq 0.001\right)$, influenced farmers' choice of methods for bird treatment. Education (odds ratio [OR] odds ratio [OR] 3.06, 95\% CI 2.10-4.44), income (OR $1.99,95 \%$ CI 1.10-3.59) and management system (OR 1.97, CI\% 1.1-3.45) were most associated with antimicrobial use. Critically important antibiotics, with lower to higher risk of antimicrobial resistance, were used by farmers (40\%). These findings showed the indiscriminate use of antimicrobials by farmers and the potential risk of antimicrobial resistance within the SPPS in Nigeria.
\end{abstract}

\section{Introduction}

The use of antimicrobials in livestock production, for disease prevention and growth promotion, is of increasing concern owing to the threat of antimicrobial resistance in both humans and animals [1]. Antimicrobial resistance has been described as one of the biggest threats to humanity, affecting critical areas such as global health, food security and livelihoods [2, 3]. Overdependence on antimicrobials by farmers, and their indiscriminate and inappropriate use are the primary divers of antimicrobial resistance [4-6]. Livestock production under intensive management system is characterized by routine use of antimicrobials, and has been associated with increased antimicrobial resistance, especially 
in poultry [7]. On the other hand, the use of antimicrobials in scavenging and semi-scavenging production systems of smallholder poultry is generally considered low due to the practice of ethnoveterinary medicine by resource-poor farmers $[8,9]$. However, recent studies have observed the increasing use of antimicrobials within the free-range, village or backyard poultry system $[4,10]$.

Also, the introduction of improved chicken breeds into smallholder poultry production systems (SPPS) as an intervention for increasing food security and livelihoods among rural households in sub-Saharan Africa [11-13] has highlighted the associated high risk of mortality in the flock due to the heterogenous condition of such environments for diseases and infection $[14,15]$. In order to prevent this risk, and increase the survivability of the improved chickens, smallholder poultry farmers are exposed to the use of antimicrobials as against adopting improved biosafety and biosecurity measures in reducing the high disease burden within the production environment $[10,16]$. Unavailability of veterinarians and animal health workers in rural communities is a contributory factor predisposing smallholder poultry farmers to indiscriminate use of antimicrobials $[1,16]$.

The improved, tropically adapted chicken breeds (FUNAAB Alpha, Noiler, Kuroiler, Sasso, ShikaBrown) were introduced to smallholder poultry farmers in Nigeria through the African Chicken Genetic Gains project (2015-2019) [11, 17]. These breeds, coupled with the existing local chicken ecotypes are a major source of animal protein (eggs, meat) in the country since smallholder poultry contribute $65-77 \%$ of the total chicken production in Nigeria [18]. This huge food resource also presents a potential risk and challenge to food safety due to the abuse of antimicrobials within the production systems. Therefore, the objective of this study was to investigate the use of antimicrobials among smallholder poultry farmers in Nigeria, as well as the prevalence of such use in relation to ethnoveterinary practices within the SPPS.

\section{Materials and Methods}

This study (cross-sectional) was conducted in five states (Rivers, Imo, Kwara, Nasarawa, Kebbi) of Nigeria each representing different agroecologies. The agroecological features of these states have been described by Yakubu et al. [19], and Bamidele and Amole [18]. A total of 350 (70 per state) smallholder poultry farmers participated in the study. The sampling method and inclusion criteria were as described by [18]. All the farmers provided informed consent and the study was approved by the Review Committee of the CGIAR COVID-19 Hub: ILRI Nigeria 2021.

Structured questionnaires were designed to provide information in the following areas: socio-demography, poultry production, disease and health management, and training in animal health. Specifically, the questionnaires elicited information on the knowledge of antimicrobial resistance, perceptions on the risks and implications of antimicrobial use, attitude of farmers towards antimicrobial usage, and the impact of COVID-19 pandemic on the procurement of antimicrobials for animal use. The questionnaires were administered to the farmers at their respective homes by trained field officers, using Google Forms accessed on smartphones. The data collection spanned 14 days (14-28 September, 2021). Data were analysed using descriptive and inferential statistics in IBM-SPSS (version 20). Data visualisations were presented using PAST (version 4.03) and Microsoft Excel (Office 2019). The antimicrobial drugs were classified based on their active ingredients and class [20], AWaRe (Access, Watch, Reserve) group list of antimicrobials [21], and WHO categorisation of importance of antibiotics in human medicine [22]. The official Central Bank of Nigeria's average monthly exchange rate used in the study was NGN 410.7 to 1 USD [23].

\section{Results}

3.1. Socio-Demographic Characteristics of Respondents. Table 1 shows that there were significant $(p<0.05)$ variations in the socio-demographic characteristics of the farmers across the five locations. Majority of the respondents were females $(71.1 \%, n=249)$, compared to the male respondents $(28.9 \%, n=101)$. There were more women (Kebbi: $77.1 \%$, Nasarawa: $90.0 \%$, Rivers: $71.4 \%$, Kwara: $67.1 \%)$ than men (Kebbi: 22.9\%, Nasarawa: 10.0\%, Rivers: 28.6\%, Kwara: $32.9 \%$ ) in all the states, except Imo (Men and women: 50\%). About one-third (30.3\%) of the respondents had no formal education while over two-thirds (69.7\%) had either primary, secondary or tertiary education. Kebbi State had the highest percentage of respondents (51.4\%) without any formal education while all the respondents (70) in Imo State had formal education. More women (89.6\%) than men (10.4\%) had zero years of schooling (106 respondents) and represents about two-fifths $(37.4 \%)$ of the female respondents. Respondents aged 48-57, and 18-27 years old had the highest (33.1\%) and lowest (3.4\%) frequency, respectively. Also, respondents who were aged 48 years old and above were about two-thirds $(64.3 \%)$ of the total number of study participants. Distribution of respondents' average monthly income shows that majority (60.6\%) of the farmers earned below NGN 30,000 ( $\$ 2.4 /$ day). The distribution of the female respondents according to the monthly earnings was $70.3 \%$ (< NGN 30,000), 25.7\% (NGN 30,000-50,000), and 4.0\% (> NGN 50,000) while that of male respondents was $36.6 \%$ (< NGN 30,000), 52.5\% (NGN 30,000-50,000) and $10.9 \%$ (>NGN 50,000).

3.2. Implication and Risks of Antimicrobial Usage. Majority (69.8\%) of the farmers did not keep records of drug use (Table 2). Imo State had the highest percentage (78.9\%) of farmers who kept records compared to Rivers (0\%), Kebbi (4.6\%), Nasarawa (22.0\%), and Kwara (39.3\%) states. Most of the farmers were not aware of the drug residue (94.3\%), withdrawal period (92.0\%), and shelf-life (expiration date) $(55.7 \%)$ associated with antimicrobial use. Farmers in Rivers (96.2\%) and Imo (98.1\%) were more aware of the shelf-life and expiration date of the drugs compared to those in other states (Kebbi: $3.1 \%$, Nasarawa: $17.1 \%$, Kwara: $32.1 \%$ ). A vast 
TABLE 1: Socio-demographic characteristics of Respondents.

\begin{tabular}{|c|c|c|c|c|c|c|c|c|}
\hline \multirow{2}{*}{ Variable } & \multicolumn{6}{|c|}{ Location } & \multirow{2}{*}{$\begin{array}{l}\text { Statistic } \\
\chi^{2}(\mathrm{df})\end{array}$} & \multirow{2}{*}{$p$ value } \\
\hline & Kebbi $n=70$ & Nasarawa $n=70$ & Rivers $n=70$ & Kwara $n=70$ & $\operatorname{Imo} n=70$ & Total $N=350$ & & \\
\hline \multicolumn{9}{|c|}{$\begin{array}{l}\text { Socio-demography } \\
\text { Gender }\end{array}$} \\
\hline Male & $16(15.8)$ & $7(6.9)$ & $20(19.8)$ & $23(22.8)$ & $35(34.7)$ & 101 & \multirow{2}{*}{$29.14(4)$} & \multirow{2}{*}{$p \leq 0.001$} \\
\hline Female & $54(21.7)$ & $63(25.3)$ & $50(20.1)$ & $47(18.9)$ & $35(14.1)$ & 249 & & \\
\hline \multicolumn{9}{|l|}{ Education } \\
\hline None & $36(34.0)$ & $20(18.9)$ & $22(20.8)$ & $28(26.4)$ & $0(0.0)$ & 106 & \multirow{4}{*}{$97.108(12)$} & \multirow{4}{*}{$p \leq 0.001$} \\
\hline Primary & $9(12.2)$ & $11(14.9)$ & $25(33.8)$ & $21(28.4)$ & $8(10.8)$ & 74 & & \\
\hline Secondary & $12(9.2)$ & $33(25.2)$ & $17(13.0)$ & $18(13.7)$ & $51(38.9)$ & 131 & & \\
\hline Tertiary & $13(33.3)$ & $6(15.4)$ & $6(15.4)$ & $3(7.7)$ & $11(28.2)$ & 39 & & \\
\hline \multicolumn{9}{|l|}{ Age group } \\
\hline $18-27$ & $7(58.3)$ & $5(41.7)$ & $0(0.0)$ & $0(0.0)$ & $0(0.0)$ & 12 & \multirow{6}{*}{$95.3(20)$} & \multirow{6}{*}{$p \leq 0.001$} \\
\hline $28-37$ & $19(42.2)$ & $17(37.8)$ & $4(8.9)$ & $0(0.0)$ & $5(11.1)$ & 45 & & \\
\hline $38-47$ & $15(22.1)$ & $15(22.1)$ & $13(19.1)$ & $13(19.1)$ & $12(17.6)$ & 68 & & \\
\hline $48-57$ & $17(14.7)$ & $16(13.8)$ & $23(19.8)$ & $38(32.8)$ & $22(19.0)$ & 116 & & \\
\hline $58-67$ & $10(10.9)$ & $17(18.5)$ & $27(29.3)$ & $18(19.6)$ & $20(21.7)$ & 92 & & \\
\hline 68 and above & $2(11.8)$ & $0(0.0)$ & $3(17.6)$ & $1(5.9)$ & $11(64.7)$ & 17 & & \\
\hline \multicolumn{9}{|l|}{ Monthly income } \\
\hline$<30,000$ & $56(26.4)$ & $60(28.3)$ & $25(11.8)$ & $50(23.6)$ & $21(9.9)$ & 212 & \multirow{3}{*}{$106.27(8)$} & \multirow{3}{*}{$p \leq 0.001$} \\
\hline $30,000-50,000$ & $11(9.4)$ & $10(8.5)$ & $30(25.6)$ & $20(17.1)$ & $46(39.3)$ & 117 & & \\
\hline$>50,000$ & $3(14.3)$ & $0(0.0)$ & $15(71.4)$ & $0(0.0)$ & $3(14.3)$ & 21 & & \\
\hline
\end{tabular}

values in parenthesis are percentages (\%).

majority (93.9\%) of the farmers neither knew if drugs consumed by chickens pass to chicken products nor if the drug residues in eggs/meat could affect humans. A greater percentage of the farmers (94.8\%) did not know if there was any risk in eating/selling eggs or meat during the administration of antimicrobials to the birds, compared with farmers (5.2\%) who indicated knowledge of such. A large proportion of the farmers were not aware of the regulations guarding antimicrobial usage (96.2\%), and were neither aware of any governing body responsible for controlling its usage $(98.2 \%)$ in livestock production. Most of the farmers (93.9\%) considered loss of flock (bird mortality) as the most important risk to avoid while considering the use of antimicrobials. The farmers were largely unaware $(93.4 \%)$ of the danger in the misuse and overuse of antimicrobials both to humans and to animals.

3.3. Description of Poultry Production and Management Systems of Respondents. The respondents' number of years of raising chickens (farming experience) ranged from 1 to 5 (16.9\%), 6-10 (37.1\%), 11-20 (29.1\%), and over 20 years (16.9\%) (Table 3). Imo (55.9\%), Kebbi (27.7\%), and Kwara $(34.3 \%)$ had the highest percentage of respondents who had been raising chickens for $0-5,6-10$, and 11-20 years, respectively. Kwara (35.6\%) and Nasarawa (30.5\%) accounted for about two-thirds $(66.1 \%)$ of the respondents who had been raising chickens for over 20 years. A vast majority $(88.9 \%)$ of the farmers had never received any training on general poultry husbandry and just over one-tenth $(11.1 \%)$ of them had ever received any training, specifically, on animal disease and health management. Location $\left(\chi^{2}=10.74, p=0.03\right)$, and not gender $\left(\chi^{2}=3.65, p=0.06\right)$ was significantly associated with respondents' training on animal disease and health management. In addition to local chickens, farmers kept improved (38.3\%) and exotic (39.4\%) chickens. The management systems practiced by the farmers were scavenging (13.7\%), semi-scavenging (49.2\%), and semi-intensive $(37.1 \%)$. Gender $\left(\chi^{2}=6.71, p=0.04\right)$ and location $\left(\chi^{2}=154.54, p \leq 0.001\right)$ were significantly associated with the type of management system. The smallholder poultry production characteristics of the farmers varied significantly $(p<0.05)$ across the locations.

3.4. Treatment Methods Used by Farmers. The treatment options adopted by farmers for treating their flock were traditional/ethnoveterinary method (39.4\%), western method (i.e. use of pharmaceuticals) (25.1\%), and a combination of both traditional and western methods (35.4\%) (Table 4). Rivers and Kwara states accounted for about onethird $(62.3 \%)$ of the farmers who only used traditional/ ethnoveterinary method while Kebbi accounted for over two-thirds (68.2\%) of the farmers who used western method. Imo and Nasarawa states had the highest percentage of farmers (32.3\%) who used both traditional and western methods. A vast majority of the farmers in Kebbi (92.9\%), Imo (74.3\%), and Nasarawa (58.6\%) states used pharmaceuticals, either alone or in combination with ethnoveterinary medicines. Gender $\left(\chi^{2}=9.30, p=0.01\right)$, location $\left(\chi^{2}=216.86, p \leq 0.01\right)$, breed-type $\left(\chi^{2}=155.92, p \leq 0.01\right)$ and management system $\left(\chi^{2}=25.08, p \leq 0.01\right)$ were significantly associated with the methods of disease treatment used by farmers. More female farmers $(43.8 \%)$ used the traditional method than the other methods (Western: 21.3\%, Both Western and Traditional: $34.9 \%)$. Most of the male farmers (36.6\%) used both traditional and western methods as against either the traditional (28.7\%) or western (34.7\%) 
TABLE 2: Farmers knowledge and awareness of the implications and risk of antimicrobials.

\begin{tabular}{|c|c|c|c|c|c|c|c|c|}
\hline \multirow[b]{2}{*}{ Questions } & \multicolumn{5}{|c|}{ Location } & \multirow[b]{2}{*}{$\begin{array}{c}\text { Total } \\
N=212\end{array}$} & \multirow[b]{2}{*}{$\chi^{2}(\mathrm{df})$} & \multirow[b]{2}{*}{$p$ value } \\
\hline & $\begin{array}{l}\text { Kebbi } \\
n=65\end{array}$ & $\begin{array}{c}\text { Nasarawa } \\
n=41\end{array}$ & $\begin{array}{l}\text { Rivers } \\
n=26\end{array}$ & $\begin{array}{l}\text { Kwara } \\
n=28\end{array}$ & $\begin{array}{c}\operatorname{Imo} \\
n=52\end{array}$ & & & \\
\hline \multicolumn{9}{|l|}{ Do you keep record of drugs used? } \\
\hline Yes & $3(4.7)$ & $9(14.0)$ & $0(0.0)$ & $11(17.2)$ & $41(64.1)$ & 64 & \multirow{2}{*}{$92.2(4)$} & \multirow{2}{*}{$p \leq 0.001$} \\
\hline No & $62(41.9)$ & $32(21.6)$ & $26(17.6)$ & $17(11.5)$ & $11(7.4)$ & 148 & & \\
\hline \multicolumn{9}{|l|}{ Are you aware of drug residue? } \\
\hline Yes & $3(25.0)$ & $0(0.0)$ & $2(16.7)$ & $3(25.0)$ & $4(33.3)$ & 12 & \multirow{2}{*}{$4.53(4)$} & \multirow{2}{*}{$p \leq 0.001$} \\
\hline No & $62(31.0)$ & $41(20.5)$ & $24(12.0)$ & $25(12.5)$ & $48(24.0)$ & 200 & & \\
\hline \multicolumn{9}{|c|}{$\begin{array}{l}\text { Are you aware of the withdrawal period when } \\
\text { using antimicrobials and do you observe it? }\end{array}$} \\
\hline Yes & $1(5.9)$ & $1(5.9)$ & $7(41.2)$ & $2(11.8)$ & $6(35.2)$ & 17 & \multirow{2}{*}{$18.93(4)$} & \multirow{2}{*}{$p \leq 0.001$} \\
\hline No & $62(32.0)$ & $40(21.0)$ & $19(10.0)$ & $26(13.0)$ & $46(24.0)$ & 195 & & \\
\hline \multicolumn{9}{|c|}{$\begin{array}{l}\text { Do you know that drugs have expiration/shelf- } \\
\text { life? }\end{array}$} \\
\hline Yes & $2(2.1)$ & $7(7.4)$ & $25(26.6)$ & $9(9.6)$ & $51(54.3)$ & 94 & \multirow{2}{*}{$148.01(4)$} & \multirow{2}{*}{$p \leq 0.001$} \\
\hline No & $63(53.3)$ & $34(28.8)$ & $1(0.9)$ & $19(16.1)$ & $1(0.9)$ & 118 & & \\
\hline \multicolumn{9}{|c|}{$\begin{array}{l}\text { Do you know if drugs taken by chickens pass to } \\
\text { the eggs/meat? }\end{array}$} \\
\hline Yes & $1(7.7)$ & $1(7.7)$ & $7(53.8)$ & $3(23.1)$ & $1(7.7)$ & 13 & \multirow{2}{*}{25.50} & \multirow{2}{*}{$p \leq 0.001$} \\
\hline No & $64(32.2)$ & $40(20.1)$ & $19(9.5)$ & $25(12.6)$ & $51(25.6)$ & 199 & & \\
\hline \multicolumn{9}{|l|}{$\begin{array}{l}\text { Do you know if drugs in eggs/meat affect } \\
\text { humans? }\end{array}$} \\
\hline Yes & $1(7.7)$ & $3(23.0)$ & $6(46.2)$ & $2(15.4)$ & $1(7.7)$ & 13 & \multirow{2}{*}{$17.10(4)$} & \multirow{2}{*}{$p \leq 0.001$} \\
\hline No & $64(32.2)$ & $38(19.1)$ & $20(10.0)$ & $26(13.1)$ & $51(25.6)$ & 199 & & \\
\hline \multicolumn{9}{|l|}{$\begin{array}{l}\text { Do you know if there is any risk in eating/ } \\
\text { Selling of eggs/meat/chicken during or } \\
\text { immediately after given drugs to the birds? }\end{array}$} \\
\hline Yes & $1(9.1)$ & $0(0.0)$ & $6(54.5)$ & $2(18.2)$ & $2(18.2)$ & 11 & \multirow{2}{*}{$21.32(4)$} & \multirow{2}{*}{$p \leq 0.001$} \\
\hline No & $64(31.8)$ & $41(20.4)$ & $20(10.0)$ & $26(12.9)$ & $50(24.9)$ & 201 & & \\
\hline \multicolumn{9}{|l|}{$\begin{array}{l}\text { Are you aware of rules/regulations of } \\
\text { antimicrobial usage? }\end{array}$} \\
\hline Yes & $1(12.5)$ & $0(0.0)$ & $3(37.5)$ & $0(0.0)$ & $4(50.0)$ & 8 & 1012 & 004 * \\
\hline No & $64(31.4)$ & $41(20.1)$ & $23(11.3)$ & $28(13.7)$ & $48(23.5)$ & 204 & $10.12(4)$ & 0.04 \\
\hline $\begin{array}{l}\text { Are you aware of any governing body } \\
\text { responsible for controlling the use of } \\
\text { antimicrobials in livestock production? }\end{array}$ & & & & & & & & \\
\hline Yes & $0(0.0)$ & $1(33.4)$ & $1(33.3)$ & $0(0.0)$ & $1(33.3)$ & 3 & 28 & 058 \\
\hline No & $65(31.1)$ & $40(19.1)$ & $25(12.0)$ & $28(13.4)$ & $51(24.4)$ & 209 & $2.84(4)$ & 0.58 \\
\hline $\begin{array}{l}\text { What do you consider as the most important } \\
\text { risk to avoid? }\end{array}$ & & & & & & & & \\
\hline Environmental pollution & $1(20.0)$ & $0(0.0)$ & $0(0.0)$ & $4(80.0)$ & $0(0.0)$ & 5 & & \\
\hline Inappropriate usage causing harm to humans & $0(0.0)$ & $6(75.0)$ & $0(0.0)$ & $2(25.0)$ & $0(0.0)$ & 8 & $40.41(8)$ & $p \leq 0.001$ \\
\hline Loss of flock (mortality) & $64(32.2)$ & $35(17.6)$ & $26(13.1)$ & $22(11.0)$ & $52(26.1)$ & 199 & & \\
\hline $\begin{array}{l}\text { Are you aware that misuse and overuse of } \\
\text { antimicrobials exposes humans and animals to } \\
\text { antimicrobial resistant bacteria with dire } \\
\text { consequence on human/animal health? }\end{array}$ & & & & & & & & \\
\hline Yes & $1(6.7)$ & $0(6.7)$ & $6(40.0)$ & $3(20.0)$ & $4(26.6)$ & 14 & & \\
\hline No & $64(32.5)$ & $41(20.3)$ & $20(10.2)$ & $25(12.7)$ & $48(24.3)$ & 198 & $17.91(4)$ & \\
\hline
\end{tabular}

${ }^{*} p<0.05$, values in parenthesis are percentages $(\%)$.

methods. The primary reason for using the traditional method by most farmers in Nasarawa (34.5\%), Rivers (43.2\%) and Kwara (40.5\%) states was affordability. Most farmers in both Kebbi (100\%) and Imo (61.1\%) states indicated the ease of availability and accessibility as their primary reason. Most of the farmers identified affordability and availability/accessibility of ethnoveterinary medicines as the primary $(38.4 \%)$ and secondary $(42.8 \%)$ reasons for the use of the traditional method.

3.5. Characteristics of Farmers' Use of Antimicrobials. A large proportion $(81.6 \%)$ of farmers who indicated using western method alone or in combination with the traditional 
TABLE 3: Features of the poultry production and management system.

\begin{tabular}{|c|c|c|c|c|c|c|c|c|}
\hline \multirow[b]{2}{*}{ Characteristics } & \multicolumn{6}{|c|}{ Location } & \multicolumn{2}{|c|}{ Statistics } \\
\hline & $\begin{array}{l}\text { Kebbi } \\
n=70\end{array}$ & $\begin{array}{c}\text { Nasarawa } \\
n=70\end{array}$ & $\begin{array}{l}\text { Rivers } \\
n=70\end{array}$ & $\begin{array}{c}\text { Kwara } \\
n=70\end{array}$ & $\begin{array}{c}\text { Imo } \\
n=70\end{array}$ & $\begin{array}{c}\text { Total } \\
N=350\end{array}$ & $\chi^{2}(\mathrm{df})$ & $p$ value \\
\hline \multicolumn{9}{|c|}{ Farming experience (years) } \\
\hline $1-5$ & $19(32.2)$ & $4(6.8)$ & $3(5.1)$ & $0(0.0)$ & $33(55.9)$ & 59 & \multirow{4}{*}{$\begin{array}{c}120.47 \\
(12)\end{array}$} & \multirow[t]{4}{*}{$p \leq 0.001$} \\
\hline $6-10$ & $36(27.7)$ & $24(18.5)$ & $34(26.2)$ & $14(10.8)$ & $22(16.9)$ & 130 & & \\
\hline $11-20$ & $11(10.8)$ & $24(23.5)$ & $24(23.5)$ & $35(34.3)$ & $8(7.8)$ & 102 & & \\
\hline$>20$ & $4(6.8)$ & $18(30.5)$ & $9(15.3)$ & $21(35.6)$ & $7(11.9)$ & 59) & & \\
\hline \multicolumn{9}{|c|}{$\begin{array}{l}\text { General training on poultry husbandry } \\
\text { and rearing }\end{array}$} \\
\hline Yes & $3(7.7)$ & $15(38.5)$ & $3(7.7)$ & $4(10.3)$ & $14(35.9)$ & 39 & \multirow{2}{*}{$21.76(4)$} & \multirow{2}{*}{$p \leq 0.001$} \\
\hline No & $67(21.5)$ & $55(17.7)$ & $67(21.5)$ & $66(21.2)$ & $56(18.0)$ & 311 & & \\
\hline \multicolumn{9}{|c|}{$\begin{array}{l}\text { Specific training on animal diseases and } \\
\text { health management }\end{array}$} \\
\hline Yes & $3(18.8)$ & $1(6.2)$ & $3(18.8)$ & $1(6.2)$ & $8(50.0)$ & 16 & \multirow{2}{*}{$10.7(4)$} & \multirow{2}{*}{$0.03^{*}$} \\
\hline No & $67(20.1)$ & $69(20.7)$ & $67(20.1)$ & $69(20.7)$ & $62(18.6)$ & 334 & & \\
\hline \multicolumn{9}{|c|}{$\begin{array}{l}\text { Type of chickens kept in addition to local } \\
\text { chickens }\end{array}$} \\
\hline Exotic & $0(0.0)$ & $33(23.9)$ & $59(42.8)$ & $12(8.7)$ & $34(24.6)$ & 138 & \multirow{3}{*}{$123.3(4)$} & \multirow{3}{*}{$p \leq 0.001$} \\
\hline Improved & $70(52.2)$ & $32(23.9)$ & $0(0.0)$ & $4(3.0)$ & $28(20.9)$ & 134 & & \\
\hline None & $0(0.0)$ & $5(6.4)$ & $11(14.1)$ & $54(69.2)$ & $8(10.3)$ & 78 & & \\
\hline \multicolumn{9}{|c|}{ Management system } \\
\hline Scavenging & $29(60.4)$ & $0(0.0)$ & $15(31.2)$ & $1(2.1)$ & $3(6.2)$ & 48 & \multirow{3}{*}{$\begin{array}{c}154.54 \\
(8)\end{array}$} & \multirow{3}{*}{$p \leq 0.001$} \\
\hline Semi-scavenging & $35(20.3)$ & $46(26.7)$ & $13(7.6)$ & $58(33.7)$ & $20(11.6)$ & 172 & & \\
\hline Semi-intensive & $6(4.6)$ & $24(18.5)$ & $42(32.3)$ & $11(8.5)$ & $47(36.2)$ & 130 & & \\
\hline
\end{tabular}

${ }^{*} p<0.05$, values in parenthesis are percentages (\%).

TABLe 4: Distribution of treatment methods available to farmers.

\begin{tabular}{|c|c|c|c|c|c|c|c|c|}
\hline \multirow[b]{2}{*}{ Characteristics } & \multicolumn{5}{|c|}{ Location } & \multirow[b]{2}{*}{$\begin{array}{c}\text { Total } \\
N=350\end{array}$} & \multirow[b]{2}{*}{$\chi^{2}(\mathrm{df})$} & \multirow[b]{2}{*}{$p$ value } \\
\hline & $\begin{array}{l}\text { Kebbi } \\
n=70\end{array}$ & $\begin{array}{c}\text { Nasarawa } \\
n=70\end{array}$ & $\begin{array}{l}\text { Rivers } \\
n=70\end{array}$ & $\begin{array}{c}\text { Kwara } \\
n=70\end{array}$ & $\begin{array}{c}\text { Imo } \\
n=70\end{array}$ & & & \\
\hline \multicolumn{9}{|l|}{ Treatment of chickens } \\
\hline Traditional & $5(3.6)$ & $29(21.0)$ & $44(32.0)$ & $42(30.4)$ & $18(13.0)$ & 138 & \multirow{3}{*}{$\begin{array}{c}216.86 \\
(8)\end{array}$} & \multirow{3}{*}{$p \leq 0.001$} \\
\hline Western & $60(68.2)$ & $1(1.1)$ & $0(0.0)$ & $15(17.1)$ & $12(13.6)$ & 88 & & \\
\hline Traditional and western & $5(4.0)$ & $40(32.3)$ & $26(21.0)$ & $13(10.5)$ & $40(32.2)$ & 124 & & \\
\hline \multicolumn{9}{|l|}{ Traditional method } \\
\hline & $n=5$ & $n=29$ & $n=44$ & $n=42$ & $n=18$ & $N=138$ & & \\
\hline \multicolumn{9}{|l|}{$\begin{array}{l}\text { Primary reason for only using } \\
\text { traditional method }\end{array}$} \\
\hline Easily administered & $0(0.0)$ & $8(36.4)$ & $4(18.2)$ & $10(45.4)$ & $0(0.0)$ & 22 & \multirow{5}{*}{$\begin{array}{c}50.35 \\
(16)\end{array}$} & \multirow{5}{*}{$p \leq 0.001$} \\
\hline Availability/accessibility & $5(12.5)$ & $7(17.5)$ & $8(20.0)$ & $9(22.5)$ & $11(27.5)$ & 40 & & \\
\hline Not costly & $0(0.0)$ & $10(18.9)$ & $19(35.8)$ & $17(32.1)$ & $7(13.2)$ & 53 & & \\
\hline Very effective & $0(0.0)$ & $1(5.3)$ & $13(68.4)$ & $5(26.3)$ & $0(0.0)$ & 19 & & \\
\hline $\begin{array}{l}\text { Safe to birds, humans and the } \\
\text { environment }\end{array}$ & $0(0.0)$ & $3(75.0)$ & $0(0.0)$ & $1(25.0)$ & $0(0.0)$ & 4 & & \\
\hline \multicolumn{9}{|l|}{ Secondary reason } \\
\hline Easily administered & $0(0.0)$ & $3(12.5)$ & $14(58.3)$ & $4(16.7)$ & $3(12.5)$ & 24 & \multirow{5}{*}{$\begin{array}{c}51.95 \\
(12)\end{array}$} & \multirow{5}{*}{$p \leq 0.001$} \\
\hline Availability/accessibility & $0(0.0)$ & $20(33.9)$ & $11(18.6)$ & $22(37.3)$ & $6(10.2)$ & 59 & & \\
\hline Not costly & $5(15.6)$ & $4(12.5)$ & $4(12.5)$ & $12(37.5)$ & $7(21.9)$ & 32 & & \\
\hline Very effective & $0(0.0)$ & $2(8.7)$ & $15(65.2)$ & $4(17.4)$ & $2(8.7)$ & 23 & & \\
\hline $\begin{array}{l}\text { Safe to birds, humans and the } \\
\text { environment }\end{array}$ & $0(0.0)$ & $0(0.0)$ & $0(0.0)$ & $0(0.0)$ & $0(0.0)$ & 0 & & \\
\hline
\end{tabular}

values in parenthesis are percentages (\%).

method, considered the cost implication of using antimicrobials on their farm. Table 5 shows that the persons who primarily influence the use of antimicrobials by farmers were veterinarian/animal health workers (27.4\%), followed by farmers' own experiences (16.0\%), local merchants of dayold and brooded chicks (15.1\%), farmer groups $(12.7 \%)$, extension agents (12.3\%), neighbours and friends (10.9\%), feed/drug seller (5.2\%), and offtakers (0.5\%). About two- 
TABLE 5: Characteristics of farmers who used antimicrobials (western method alone, or in combination with traditional method).

\begin{tabular}{|c|c|c|c|c|c|c|c|c|}
\hline \multirow[b]{2}{*}{ Characteristics } & \multicolumn{5}{|c|}{ Location } & \multirow[b]{2}{*}{$\begin{array}{c}\text { Total } \\
N=212\end{array}$} & \multirow[b]{2}{*}{$\chi^{2}(\mathrm{df})$} & \multirow[b]{2}{*}{$p$ value } \\
\hline & $\begin{array}{l}\text { Kebbi } \\
n=65\end{array}$ & $\begin{array}{c}\text { Nasarawa } \\
n=41\end{array}$ & $\begin{array}{l}\text { Rivers } \\
n=26\end{array}$ & $\begin{array}{l}\text { Kwara } \\
n=28\end{array}$ & $\begin{array}{c}\operatorname{Imo} \\
n=52\end{array}$ & & & \\
\hline \multicolumn{9}{|l|}{$\begin{array}{l}\text { Primary influence on the decision to use } \\
\text { antimicrobials }\end{array}$} \\
\hline Farmer group & $0(0.0)$ & $3(11.1)$ & $15(55.6)$ & $0(0.0)$ & $9(33.3)$ & 27 & \multirow{8}{*}{$\begin{array}{c}320.19 \\
(28)\end{array}$} & \multirow{8}{*}{$p \leq 0.001$} \\
\hline Feed/drug seller & $0(0.0)$ & $0(0.0)$ & $1(9.1)$ & $0(0.0)$ & $10(90.9)$ & 11 & & \\
\hline Local merchants (day-old/brooded chicks) & $20(62.5)$ & $2(6.2)$ & $0(0.0)$ & $10(31.2)$ & $0(0.0)$ & 32 & & \\
\hline Neighbors/friend & $1(4.3)$ & $3(13.0)$ & $3(13.0)$ & $7(30.4)$ & $9(39.1)$ & 23 & & \\
\hline Veterinarian/animal health worker & $44(75.9)$ & $4(6.9)$ & $1(1.7)$ & $3(5.2)$ & $6(10.3)$ & 58 & & \\
\hline Own experiences & $0(0.0)$ & $3(8.8)$ & $6(17.6)$ & $8(23.5)$ & $17(50.0)$ & 34 & & \\
\hline Offtakers & $0(0.0)$ & $0(0.0)$ & $0(0.0)$ & $0(0.0)$ & $1(100)$ & 1 & & \\
\hline Extension agent & $0(0.0)$ & $26(100.0)$ & $0(0.0)$ & $0(0.0)$ & $0(0.0)$ & 26 & & \\
\hline \multicolumn{9}{|l|}{ Secondary influence } \\
\hline Farmer group & $5(23.8)$ & $0(0.0)$ & $5(23.8)$ & $0(0.0)$ & $11(52.4)$ & 21 & \multirow{8}{*}{$\begin{array}{c}163.5 \\
(28)\end{array}$} & \multirow{8}{*}{$p \leq 0.001$} \\
\hline Feed/drug seller & $6(15.4)$ & $1(2.6)$ & $9(23.1)$ & $2(5.1)$ & $21(53.8)$ & 39 & & \\
\hline Local merchants (day-old/brooded chicks) & $10(100)$ & $0(0.0)$ & $0(0.0)$ & $0(0.0)$ & $0(0.0)$ & 10 & & \\
\hline Neighbors/friend & $7(14.6)$ & $16(33.3)$ & $7(14.6)$ & $9(18.8)$ & $9(18.8)$ & 48 & & \\
\hline Veterinarian/animal health worker & $33(75.0)$ & $3(6.8)$ & $5(11.4)$ & $3(6.8)$ & $0(0.0)$ & 44 & & \\
\hline Own experiences & $3(7.0)$ & $18(41.9)$ & $0(0.0)$ & $13(30.2)$ & $9(20.9)$ & 43 & & \\
\hline Offtakers & $0(0.0)$ & $3(75.0)$ & $0(0.0)$ & $1(0.25)$ & $0(0.0)$ & 4 & & \\
\hline Extension agent & $1(33.3)$ & $0(0.0)$ & $0(0.0)$ & $0(0.0)$ & $2(66.7)$ & 3 & & \\
\hline \multicolumn{9}{|l|}{$\begin{array}{l}\text { What has the most influence on the use of } \\
\text { antimicrobials? }\end{array}$} \\
\hline Birds are dying & $21(39.6)$ & $0(0.0)$ & $21(39.6)$ & $4(7.6)$ & $7(13.2)$ & 53 & \multirow{5}{*}{$\begin{array}{c}83.69 \\
(16)\end{array}$} & \multirow{5}{*}{$p \leq 0.001$} \\
\hline $\begin{array}{l}\text { The moment birds show any sign and } \\
\text { symptom of disease }\end{array}$ & $34(28.1)$ & $32(26.4)$ & $1(0.8)$ & $14(11.6)$ & $40(33.1)$ & 121 & & \\
\hline Make birds eat/grow more & $0(0.0)$ & $0(0.0)$ & $1(100)$ & $0(0.0)$ & $0(0.0)$ & 1 & & \\
\hline Prevent sickness & $10(31.3)$ & $9(28.0)$ & $3(9.4)$ & $10(31.3)$ & $0(0.0)$ & 32 & & \\
\hline $\begin{array}{l}\text { Prevent wastage of antimicrobials drug } \\
\text { that is about to expire }\end{array}$ & $0(0.0)$ & $0(0.0)$ & $0(0.0)$ & $0(0.0)$ & $5(0.0)$ & 5 & & \\
\hline \multicolumn{9}{|l|}{ Primary purpose of antimicrobials use } \\
\hline Treatment & $10(100)$ & $0(0.0)$ & $0(0.0)$ & $0(0.0)$ & $0(0.0)$ & 10 & \multirow{3}{*}{$49.35(8)$} & \multirow{3}{*}{$p \leq 0.001$} \\
\hline Prevention & $11(30.6)$ & $1(2.8)$ & $12(33.3)$ & $7(19.4)$ & $5(13.9)$ & 36 & & \\
\hline Treatment and prevention & $44(26.5)$ & $40(24.1)$ & $14(8.4)$ & $21(12.7)$ & $47(28.3)$ & 166 & & \\
\hline \multicolumn{9}{|l|}{ Source of prescription } \\
\hline Extension agent & $56(51.4)$ & $39(35.8)$ & $0(0.0)$ & $2(1.8)$ & $12(11.0)$ & 109 & \multirow{3}{*}{$\begin{array}{l}173.98 \\
(8)\end{array}$} & \\
\hline Self-prescription & $5(7.1)$ & $1(1.4)$ & $11(15.5)$ & $15(21.1)$ & $39(54.9)$ & 71 & & $p \leq 0.001$ \\
\hline Veterinarian/animal health worker & $4(12.5)$ & $1(3.1)$ & $15(46.9)$ & $11(34.4)$ & $1(3.1)$ & 32 & & \\
\hline Source of drugs & & & & & & & & \\
\hline Feedstore & $3(9.6)$ & $25(80.7)$ & $0(0.0)$ & $1(3.2)$ & $2(6.5)$ & 31 & & \\
\hline Local vendor & $43(67.2)$ & $1(1.6)$ & $0(0.0)$ & $5(7.8)$ & $15(23.4)$ & 64 & 209.25 & \\
\hline Pharmacy/Chemist & $0(0.0)$ & $0(0.0)$ & $6(13.3)$ & $8(17.8)$ & $31(68.9)$ & 45 & $(12)$ & $p \leq 0.001$ \\
\hline Veterinary drug store & $19(26.4)$ & $15(20.8)$ & $20(27.8)$ & $14(19.4)$ & $4(5.6)$ & 72 & & \\
\hline $\begin{array}{l}\text { Has the COVID-19 pandemic affected how } \\
\text { you source for these drugs? }\end{array}$ & & & & & & & & \\
\hline Yes & $59(67.0)$ & $25(28.4)$ & $4(4.5)$ & $0(0.0)$ & $0(0.0)$ & 88 & & \\
\hline No & $5(5.4)$ & $11(11.8)$ & $9(9.7)$ & $28(30.1)$ & $40(43.0)$ & 93 & 171.56 & $p \leq 0.001$ \\
\hline Not sure & $1(3.2)$ & $5(16.1)$ & $13(41.9)$ & $0(0.0)$ & $12(38.7)$ & 31 & & \\
\hline Route of administration & & & & & & & & \\
\hline Food & $0(0.0)$ & $0(0.0)$ & $1(11.1)$ & $0(0.0)$ & $8(88.9)$ & 9 & & \\
\hline Water & $50(44.6)$ & $5(4.5)$ & $21(18.8)$ & $27(24.1)$ & $9(8.0)$ & 112 & & \\
\hline Food and water & $15(16.5)$ & $36(39.6)$ & $4(4.4)$ & $1(1.0)$ & $35(38.5)$ & 91 & $(12)$ & $p \leq 0.001$ \\
\hline Injection & $0(0.0)$ & $0(0.0)$ & $0(0.0)$ & $0(0.0)$ & $0(0.0)$ & 0 & & \\
\hline Pattern of usage & & & & & & & & \\
\hline 1 antimicrobial & $50(34.3)$ & $39(26.7)$ & $26(17.8)$ & $13(8.9)$ & $18(12.3)$ & 146 & & \\
\hline$\geq 2$ antimicrobials & $15(22.7)$ & $2(3.1)$ & $0(0.0)$ & $15(22.7)$ & $34(51.5)$ & 66 & $61.93(4)$ & $p \leq 0.001$ \\
\hline
\end{tabular}


TABLE 5: Continued.

\begin{tabular}{|c|c|c|c|c|c|c|c|c|}
\hline \multirow[b]{2}{*}{ Characteristics } & \multicolumn{5}{|c|}{ Location } & \multirow[b]{2}{*}{$\begin{array}{c}\text { Total } \\
N=212\end{array}$} & \multirow[b]{2}{*}{$\chi^{2}(\mathrm{df})$} & \multirow[b]{2}{*}{$p$ value } \\
\hline & $\begin{array}{l}\text { Kebbi } \\
n=65\end{array}$ & $\begin{array}{c}\text { Nasarawa } \\
n=41\end{array}$ & $\begin{array}{l}\text { Rivers } \\
n=26\end{array}$ & $\begin{array}{c}\text { Kwara } \\
n=28\end{array}$ & $\begin{array}{c}\text { Imo } \\
n=52\end{array}$ & & & \\
\hline Occasionally & $65(35.0)$ & $37(19.9)$ & $25(13.4)$ & $11(5.9)$ & $48(25.8)$ & 186 & & \\
\hline Seldomly & $0(0.0)$ & $4(57.1)$ & $1(14.3)$ & $1(14.3)$ & $1(14.3)$ & 7 & 101.56 & $p \leq 0.001$ \\
\hline Regularly & $0(0.0)$ & $0(0.0)$ & $0(0.0)$ & $16(84.2)$ & $3(15.8)$ & 19 & & \\
\hline \multicolumn{9}{|c|}{$\begin{array}{l}\text { Do you complete the required treatment } \\
\text { dosage? }\end{array}$} \\
\hline Yes & $27(23.5)$ & $33(28.7)$ & $25(21.7)$ & $14(12.2)$ & $16(13.9)$ & 115 & & \\
\hline No & $33(52.4)$ & $0(0.0)$ & $0(0.0)$ & $1(1.6)$ & $29(46.0)$ & 63 & $90.74(8)$ & $p \leq 0.001$ \\
\hline Does not know there is a dosage & $5(14.7)$ & $8(23.5)$ & $1(3.0)$ & $13(38.2)$ & $7(20.6)$ & 34 & & \\
\hline \multicolumn{9}{|l|}{ Storage of antimicrobials } \\
\hline Poultry shed & $2(22.2)$ & $5(55.6)$ & $0(0.0)$ & $1(11.1)$ & $1(11.1)$ & 9 & & \\
\hline Refrigerator & $0(0.0)$ & $1(6.2)$ & $15(93.8)$ & $0(0.0)$ & $0(0.0)$ & 16 & 120.5 & \\
\hline Somewhere in the house & $63(34.1)$ & $35(18.9)$ & $11(5.9)$ & $27(14.6)$ & $49(26.5)$ & 185 & $4(12)$ & -0.0 \\
\hline Outside the house & $0(0.0)$ & $0(0.0)$ & $0(0.0)$ & $0(0.0)$ & $2(100.0)$ & 2 & & \\
\hline \multicolumn{9}{|c|}{$\begin{array}{l}\text { Do you know the name of the antimicrobials } \\
\text { used? }\end{array}$} \\
\hline Yes & $6(8.9)$ & $11(16.4)$ & $2(3.0)$ & $2(3.0)$ & $46(68.7)$ & 67 & & \\
\hline No & $49(50.5)$ & $12(12.4)$ & $21(21.6)$ & $11(11.3)$ & $4(4.12)$ & 97 & $\begin{array}{c}143.44 \\
(8)\end{array}$ & $p \leq 0.001$ \\
\hline No, but I can describe it & $10(20.8)$ & $18(37.5)$ & $3(6.2)$ & $5(31.3)$ & $2(4.2)$ & 48 & & \\
\hline
\end{tabular}

values in parenthesis are percentages (\%).

thirds $(63.7 \%)$ of the farmers signified the influence of neighbours and friends $(22.6 \%)$, veterinarian/animal health workers $(20.8 \%)$, and own experiences $(20.3 \%)$ as the secondary reasons for the use of antimicrobials. Over half $(57.1 \%)$ of the farmers indicated "the moment the birds begin to show signs and symptoms of any disease" as the most important factor influencing the use of antimicrobials. A vast majority (78.3\%) of the farmers used antimicrobials for both prevention and treatment. Some of the farmers obtained their prescription from extension agents (51.4\%) and veterinarians/animal health worker $(15.1 \%)$ while onethird $(33.5 \%)$ use self-prescription. The farmers purchased the antimicrobials from feedstores (14.6\%), local vendors (30.2\%), pharmacies/chemists (21.2\%), and veterinary stores (34.0\%). Majority (95.5\%) of the farmers whose sourcing of antimicrobials was impacted by COVID-19 pandemic were from Kebbi $(91.0 \%)$ and Nasarawa $(61.0 \%)$ states. The main routes of drug administration by the farmers were water $(52.8 \%)$, food $(4.3 \%)$, food and water $(42.9 \%)$. Over twothirds $(68.9 \%)$ of the farmers used only one type of antimicrobials at a time compared to about one-third $(31.1 \%)$ of the farmers who used at least two types. Gender $\left(\chi^{2}=5.64\right.$, $p=0.02)$ and location $\left(\chi^{2}=61.93, p \leq 0.001\right)$ significantly influenced the pattern of antimicrobials usage. More women used one type $(71.2 \%)$ or at least two types $(54.6 \%)$ of antimicrobials compared to men $(1: 28.8 \%, \geq 2: 45.4 \%)$.

Kwara $(22.7 \%)$ and Imo (51.5\%) accounted for most (74.2\%) of the farmers who used more than two types of antimicrobials. Farmers used antimicrobials occasionally (infrequent/irregular intervals) (87.7\%), seldomly (i.e. rarely, when the local medicines do not seem to be effective) (3.3\%), and regularly (once per week/month) (9.0\%). Most (84.2\%) of the farmers in Kwara State used antimicrobials more regularly than the other states. Gender $\left(\chi^{2}=6.30, p=0.04\right)$ and location $\left(\chi^{2}=101.56, p \leq 0.001\right)$ significantly influenced the frequency of antimicrobials usage. More men (57.9\%) used antimicrobials regularly than women (42.1\%). More women described their use of antimicrobials as occasionally (67.7\%) and seldomly (85.7\%) compared to men (occasionally: $32.3 \%$, seldomly: $14.3 \%)$.

Over half $(54.3 \%)$ of the farmers completed the required dosage during treatment compared to those (29.7\%) who did not complete the dosage. Kebbi (50.0\%) and Imo (46.0\%) states accounted for most (98.4\%) of the farmers who do not complete the required treatment dosage. Over one-tenth $(16.0 \%)$ of the farmers did not know there was a required dosage for antimicrobials usage. A vast majority (87.3\%) of the farmers stored the drugs somewhere in the house, with only a few (7.6\%) using refrigerator. About one-third $(31.6 \%)$ of the farmers knew the names of the antimicrobials administered. Most of the farmers (45.8\%) did not know the names of the antimicrobials given to the birds but about onefourth $(22.6 \%)$ of these farmers could describe the antimicrobials. Figure 1 shows the antimicrobial drugs commonly used by the farmers. All the antimicrobials, except oxytetracycline (watch list) were on the access group list of antimicrobials [20]. Tetracycline was the most commonly used antimicrobial drug by majority of the farmers $(41.0 \%)$ while Keproceryl ${ }^{\circledR}$ (a mix of oxytetracycline, erythromycin, colistin and streptomycin) and amoxicillin were the least used antimicrobial drug by farmers (1.0\%). Clustering of the drugs based on the similarity index for the different classes of antimicrobials showed the following clusters: Tetracycline and oxytetracycline (Tetracyclines), Amprocox ${ }^{\circledR}$ (Amprolium + sulphaquinoxaline sodium) and Septrin ${ }^{\circledR / \text { co-tri- }}$ moxazole (Sulfonamides), amoxicillin, ampicillin and Ampiclox ${ }^{\circledR}$ (Ampicillin + Cloxacillin) (Penicillins), chloramphenicol (Amphenicols), Flagyl ${ }^{\circledR} /$ metronidazole $^{2}$ 


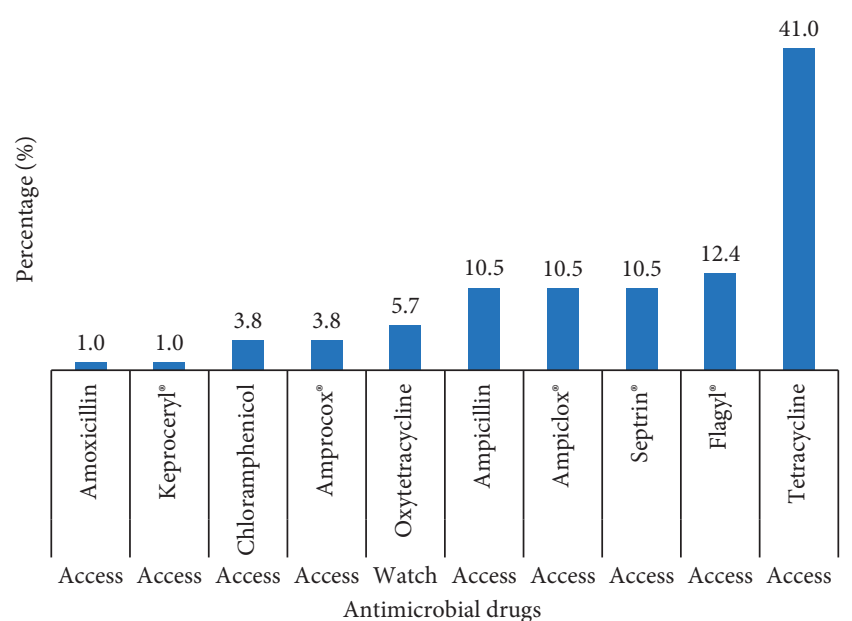

Figure 1: Antimicrobial drugs commonly used by farmers. Tetracycline is the most commonly used antimicrobial drug $(n=212)$.

(imidazole), and Keproceryl ${ }^{\circledR}$ (Aminoglycosides) (Figure 2). Further categorisation of the antimicrobials, based on the risk to human health was as follows: critically important (40\%) (Ampiclox ${ }^{\circledR}$, Keproceryl $^{\circledR}$, amoxicillin, ampicillin), highly important $(37 \%)$ (Amprocox ${ }^{\circledR}$, Septrin ${ }^{\circledR}$, chloramphenicol, tetracycline, oxytetracycline), and important (23\%) (Flagyl ${ }^{\circledR}$ ) (Figure 3). The critically important antibiotic drugs were mostly used by farmers in Imo State (81.0\%) compared to the other states (Nasarawa: 14.3\%, Kebbi: 4.8\%, Rivers and Kwara: 0\%). In comparison with other states (Kebbi:0 and 9.4\%, Nasarawa 7.1 and 17.2\%, Rivers 7.1 and 1.6\%, Kwara: 7.1 and 3.1\%), Imo State represented the highest users of both the important (78.6\%) and highly important (68.8\%) antibiotic drugs. There were more women (Important: 64.3\%, Highly important: 54.7\%, Critically important: $71.4 \%)$ than men using all the three categories of antibiotics. Gender $(\chi 2=1.99, p=0.36)$ and location $(\chi 2=6.54, p=0.59)$ had no significant influence on antibiotics categorisation among the farmers.

3.6. Factors Associated with the Use of Antimicrobials. Table 6 highlights the influence of the independent factors (location, gender, education, age, income, farming experience, and management system) in predicting antimicrobial usage. The model shows that the factors were good predictors $(\beta=0.429, \mathrm{df}=1, p<0.05)$ of antimicrobials use among the farmers. Educational level, family income, management system, age, location, and breed-type were $3.055,1.987,1.965$, $0.741,0.510$, and 0.398 times more likely $(p<0.05)$ to influence farmers' use of antimicrobials, respectively. Gender, and farmers' years of keeping chicken did not have a statistically significant effect $(p>0.05)$ on the use of antimicrobials.

\section{Discussion}

Globally, the indiscriminate use of antibiotics in poultry production is a driver of antimicrobial resistance within the food chain. Antimicrobial use in smallholder poultry production in Nigeria is of particular importance due to the

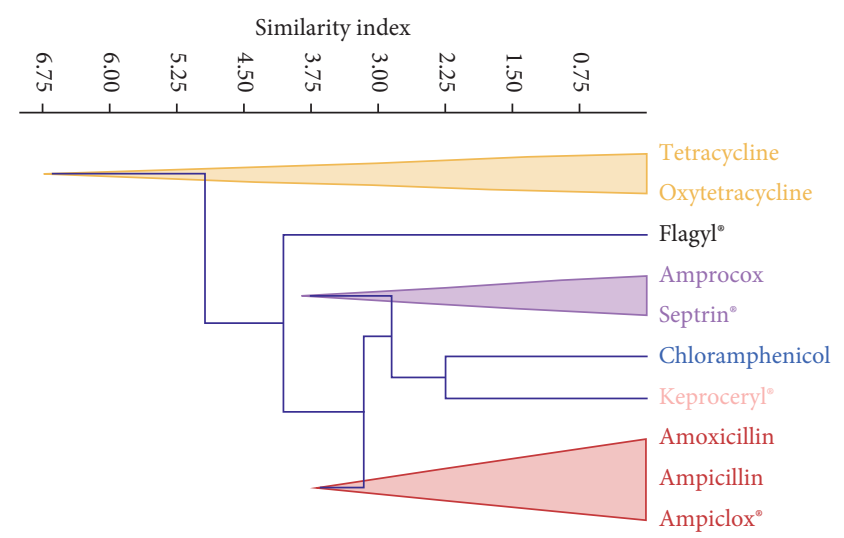

Figure 2: Cluster plot of the antimicrobial drugs used by farmers. Antimicrobials clustered as tetracyclines (tetracycline and oxytetracycline), sulfonamides (amprocox ${ }^{\circledR}$ and septrin ${ }^{\circledR}$ ), penicillins (amoxicillin, ampicillin and ampiclox ${ }^{\circledR}$ ), amphenicols chloramphenicol, and aminoglycosides (Keproceryl ${ }^{\circledR}$ ).

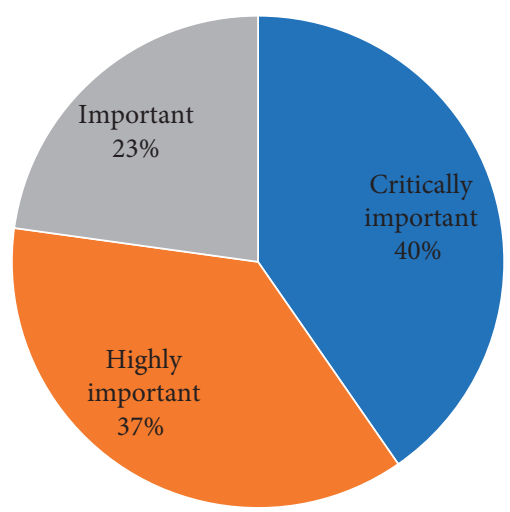

FIGURE 3: Categorisation of farmers' use of antimicrobials based on the WHO criteria for ranking antimicrobial drug use in human medicine $(n=212)$.

increasing shift in consumer preference for organicallyraised village chickens (local, improved) produced under scavenging and semi-scavenging production systems. Chickens reared under such systems have been reported to have a lower risk of antimicrobial resistance $[23,24]$ because women who are the primary keepers of these birds, also serve as custodians of the indigenous veterinary knowledge (ethnoveterinary medicine) used in the treatment of diseases and general flock management $[8,10,25,26]$. The result of this study agrees with previous studies on the role and dominance of women in smallholder poultry production in developing countries [26-28]. Compared to men (10.9\%), over one-third $(37.4 \%)$ of the women $(37.4 \%)$ sampled in this study were uneducated ( 0 years of schooling). The high illiteracy observed among women limits the technical efficiency, productivity and performance of the primary chicken producers within the smallholder poultry value chain [29-31]. Specifically, it limits the capacity to adequately administer, monitor and keep records of antimicrobial use on the farm. This is supported by our findings on the record keeping, handling, storage and administration of antimicrobials by farmers sampled in this study. 
TABLE 6: Distribution of independent variables predicting antimicrobial usage in smallholder poultry production.

\begin{tabular}{|c|c|c|c|c|c|c|c|c|}
\hline \multirow{2}{*}{ Dependent variable: The use of antibiotics } & \multirow{2}{*}{$\beta$} & \multirow{2}{*}{ SE } & \multirow{2}{*}{ Wald statistics } & \multirow{2}{*}{ Df } & \multirow{2}{*}{$p$ value } & \multirow{2}{*}{ OR } & \multicolumn{2}{|c|}{ C.I. (OR) } \\
\hline & & & & & & & Lower & Upper \\
\hline Constant & 1.115 & 0.993 & 1.261 & 1 & 0.261 & 3.048 & & \\
\hline Location & -0.673 & 0.129 & 27.078 & 1 & $0.000^{*}$ & 0.510 & 0.396 & 0.657 \\
\hline Gender & -0.389 & 0.343 & 1.291 & 1 & 0.256 & 0.678 & 0.346 & 1.326 \\
\hline Education & 1.117 & 0.191 & 34.246 & 1 & $0.000^{*}$ & 3.055 & 2.102 & 4.440 \\
\hline Age & -0.300 & 0.151 & 3.952 & 1 & $0.047^{*}$ & 0.741 & 0.551 & 0.996 \\
\hline Family income & 0.687 & 0.301 & 5.207 & 1 & $0.023^{*}$ & 1.987 & 1.102 & 3.585 \\
\hline Years of keeping chickens & -0.150 & 0.167 & 0.802 & 1 & 0.371 & 0.861 & 0.621 & 1.195 \\
\hline Breed type & -0.922 & 0.391 & 5.567 & 1 & $0.018^{*}$ & 0.398 & 0.185 & 0.855 \\
\hline Management system & 0.676 & 0.287 & 5.532 & 1 & $0.019^{*}$ & 1.965 & 1.119 & 3.452 \\
\hline
\end{tabular}

${ }^{*} p<0.05, \beta$ : beta coefficient, SE: standard error, Df: degree of freedom, OR: odds ratio, CI: confidence interval (95\%).

High illiteracy among women also suggests that the women are innumerate. This portends a high possibility for the indiscriminate use of antimicrobials by resource-poor women, majority $(70.3 \%)$ of whom earned below NGN 30,000 per month (i.e. USD 2.4/person/day). This is consistent with the overall poor outlook of women incomes and lower wages compared to men in smallholder farming [32]. Also, in a recent study, Bamidele and Amole [18] reported lower monthly incomes for women chicken producers in Nigeria compared to men.

Over half (54.0\%) of the farmers had between 0 and 10 years' experience keeping smallholder poultry. This is similar to the number of years of farming experience reported by Alemayehu et al. [33], but is at variance with the report by $\mathrm{Xu}$ et al. [34] who observed that most farmers in Northwestern China had between 10 and 19 years farming experience. Despite the high percentage of farmers $(83.1 \%)$ with over 5 years farming experience, a large proportion $(88.9 \%)$ of the farmers had never been trained on poultry keeping, and only a few $(11.1 \%)$ had received a form of training on animal health. Lack of basic training in husbandry and health management is a characteristic description of smallholder farming systems because farmers' access to quality training by subject-matter specialists and agricultural extension agents is poor and limited in rural communities [34, 35]. Keeping of improved and exotic chickens under the semi-scavenging and semi-intensive systems of production requires farmers to be adequately trained and informed on the management (housing, nutrition, health, biosecurity) skills applicable under such production systems [31]. Most farmers (39.4\%) in this study, especially women practiced ethnoveterinary medicine (traditional method) for disease treatment and health management. This was most common with farmers who only kept local chickens (53.6\%) as against those who reared exotic (42.0\%) and improved (4.4\%) chickens in addition to the local chicken ecotypes. Compared with the scavenging $(16.0 \%)$ and semi-intensive (30.4\%) production systems, the semi-scavenging system of production had the highest percentage $(53.6 \%)$ of farmers applying the indigenous veterinary knowledge for bird treatment. Affordability (low cost) $(38.4 \%)$ and availability (42.8\%) were identified as the highest primary and secondary factors influencing the use of ethnoveterinary medicine, respectively. Previous studies have equally identified accessibility and low cost as the main drivers of ethnoveterinary medicine among farmers in SPPS $[26,36,37]$. Unsurprisingly, animal welfare, food safety and environmental considerations were the least factors motivating the use of traditional and local medicines on smallholder poultry farms.

Over two-thirds (35.4\%) of the farmers were observed to be using a combination of ethnoveterinary and western methods of treatment in disease treatment and health management. This practice was common with farmers keeping exotic chickens (50.8\%) in addition to local chickens than with those who kept improved (33.1\%) breeds. It was least common with farmers who only kept local chickens (16.1\%). While low cost of ethnoveterinary medicine was a major driver for its application, farmers who solely used western methods of pharmaceuticals treatment or in combination with traditional local herbs, indicated the cost implication of antimicrobial administration on the overall production and profitability of the farm enterprise. However, it has been reported that the cost of antimicrobials is relatively low and its use does not affect the profitability and economics of production in smallholdings $[38,39]$. Administration of the antimicrobial drugs was mostly through water, a route previously reported as being the most used by poultry farmers [40-42].

This study observed a strong association between the locations (agroecology) and the various factors (primary and secondary) influencing the use of antimicrobials by farmers. These findings are in consonance with the reported intricate linkage between farmers' use of antimicrobials and veterinarians, poultry dealers and merchants, and sales representatives of feed companies and pharmaceuticals [43]. Our findings show that the behavioural tendency of farmers towards using antimicrobials is driven by the onset of any sign of illness in the birds, even though most of the farmers have not received any formal training on the identification of diseases and animal health management. This behaviour may explain the observed high percentage of farmers who indulge in self-prescription based on self-diagnosis, consequently risking an incorrect diagnosis and wrong use of antimicrobial drugs [44].

As previously reported by other studies for both commercial poultry (intensive) and small-scale poultry [32, 43-45], our findings also show multiple antimicrobial 
drug usage in SPPS. Multi-drug use was most common in semi-intensive production systems $(69.7 \%)$ than in the other two systems (scavenging: 6.1\%, Semi-scavenging: $24.2 \%$ ), and was common with farmers who kept exotic (53.0\%) and improved (33.3\%) breeds. The high prevalence of multi-drug use observed in this study is similar to that previously reported for commercial and intensively managed farms in both developed and developing countries [32, 41, 46]. Unlike in developed nations, where policies have been enacted to prevent indiscriminate use of antimicrobials in livestock production, the ease of purchasing over-the-counter antimicrobial drugs, lack of training, and poor regulation of veterinary and animal practices are some of the factors fueling multi-drug use in the developing countries [6, 47]. Tetracycline in combination with either Flagyl ${ }^{\circledR}(24.2 \%)$ or Ampiclox $^{\circledR}(18.2 \%)$ were the most predominant multi-drug used by farmers in this study. Tetracycline is reportedly one of the most commonly used antibiotics drug class in Africa $[40,43,48]$.

All the antimicrobials were on the access list of antibiotic drug groups, except oxytetracyline, which was on the watch list. According to WHO [21], antibiotics on the access and watch lists have lower and higher resistance potentials than those on the reserve list (highest potential), respectively. This study identified the presence of the critically important (4 classes), highly important (5 classes), and important (1 class) antibiotic drugs used in human medicine [22]. The critically important antibiotic drugs were used by a larger proportion of the farmers (40\%), than the other two classes. The presence of critically important antibiotics, with lower to higher resistance potential presents a potential public health threat, of antimicrobial resistance, to Nigerians who are increasingly demanding for organically-raised village chickens in place of the intensively-produced poultry products [49-51]. Our finding is consistent with the ranking of antimicrobial drug categorisation in Bangladesh, a country with similar economic and agricultural status as Nigeria [45].

The knowledge and awareness of farmers on the conditions (shelf-life, storage, withdrawal period, drug residue) for antimicrobial usage and the associated risks to humans, animals and the environment was poor. In addition to age and educational level, location, as a description of the agroecological zones was a significant predictor of antimicrobial usage among smallholder poultry farmers in Nigeria.

\section{Conclusion}

To the best of our knowledge, this study is the first to report on antimicrobial usage in SPPS across major agroecological zones of Nigeria. Most farmers still practice ethnoveterinary medicine. Antimicrobial drugs were used by farmers who kept both improved (FUNAAB Alpha, Noiler, Sasso, Kuroiler, ShikaBrown) and exotic (Broiler and layers) birds in addition to the local chicken ecotypes. Education, income and management system had the highest of influence on antimicrobial use. Multi-drug use was prevalent in the semiintensive system of production with tetracycline being the drug mostly administered in combination with Flagyl ${ }^{\circledR}$ or
Ampiclox ${ }^{\circledR}$. There is a high risk of indiscriminate use of antimicrobial drugs within SPPS in Nigeria because of the high level of illiteracy observed among the women, who are the primary producers of village chickens. The use of critically important antibiotics threatens the consumption of poultry products, and presents smallholder poultry as a reservoir for antimicrobial resistance in humans. Provision of specialized trainings on animal disease and health management among smallholder poultry farmers will improve farmers' knowledge and awareness of antimicrobial resistance and stem the tide of antimicrobial drug abuse in livestock production.

\section{Data Availability}

Data are available on request from the corresponding author. After institutional review, all data will be publicly available on the ILRI dataset portal at https://data.ilri.org/ portal/.

\section{Conflicts of Interest}

The authors declare there are no conflicts of interest regarding the publication of this paper.

\section{Acknowledgments}

The authors express their appreciation to all the farmers who consented to participate in the study. The authors recognize the efforts of the field officers during the survey and are grateful to the staff of ILRI, Ibadan, for their assistance during the entire study period. This research was funded by the CGIAR Research Program for Agriculture for Nutrition and Health (A4NH) as part of the CGIAR COVID-19 Hub project in Nigeria.

\section{References}

[1] Magnusson, How to Use Anitibiotics Effectively and Responsibly in Poultry Production-for the Sake of Human and Animal Health, FAO, Budapest, Hungary, 2021.

[2] WHO, Antimicrobial Resistance: Global Report on Surveillance, WHO, Geneva, Switzerland, 2014.

[3] WHO, Antimicrobial Resistance, WHO, Geneva, Switzerland, 2021, https://www.who.int/news-room/fact-sheets/detail/ antimicrobial-resistance.

[4] L. Glasgow, M. Forde, D. Brow, C. Mahoney, S. Fletcher, and S. Rodrigo, "Antibiotic use in poultry production in Grenada," Veterinary Medicine International, vol. 2019, Article ID 6785195, 7 pages, 2019.

[5] T. T. H. Van, Z. Yidana, P. M. Smooker, and P. J. Coloe, "Antibiotic use in food animals worldwide, with a focus on Africa: pluses and minuses," Journal of Global Antimicrobial Resistance, vol. 20, pp. 170-177, 2020.

[6] K. Geta and M. Kibret, "Knowledge, attitudes and practices of animal farm owners/workers on antibiotic use and resistance in Amhara region, north western Ethiopia," Scientific Reports, vol. 11, no. 1, pp. 1-13, 2021.

[7] I. Chantziaras, F. Boyen, B. Callens, and J. Dewulf, "Correlation between veterinary antimicrobial use and antimicrobial resistance in food-producing animals: a report on seven 
countries," Journal of Antimicrobial Chemotherapy, vol. 69, no. 3, pp. 827-834, 2014.

[8] E. F. Guèye, "Ethnoveterinary medicine against poultry diseases in African villages," World's Poultry Science Journal, vol. 55, no. 2, pp. 187-198, 1999.

[9] D. A. Ekunseitan, M. A. Adeyemi, S. S. Abiola, O. O. Oluwatosin, O. M. Sogunle, and E. Fabusoro, "Perception of ethno-veterinary practices in selected villages in Ogun state," Nigerian Journal of Animal Science, vol. 2016, no. 1, pp. 108-127, 2016.

[10] E. F. Gueye, "Newcastle disease in family poultry: prospects for its control through ethnoveterinary medicine," Livestock Research for Rural Development, vol. 14, no. 5, pp. 80-91, 2002.

[11] N. B. Alhaji, A. E. Haruna, B. Muhammad, M. K. Lawan, and T. O. Isola, "Antimicrobials usage assessments in commercial poultry and local birds in North-Central Nigeria: associated pathways and factors for resistance emergence and spread," Preventive Veterinary Medicine, vol. 154, pp. 139-147, 2018.

[12] F. O. Ajayi, O. Bamidele, W. A. Hassan et al., "Production performance and survivability of six dual-purpose breeds of chicken under smallholder farmers' management practices in Nigeria," Archives of Animal Breeding, vol. 63, no. 2, pp. 387-408, 2020.

[13] F. S. Guni, S. H. Mbaga, A. M. Katule, and E. H. Goromela, "Performance evaluation of Kuroiler and Sasso chicken breeds reared under farmer management conditions in highland and lowland areas of Mvomero district, Eastern Tanzania," Tropical Animal Health and Production, vol. 53, no. 2, pp. 53-59, 2021.

[14] S. Abegaz, W. Esatu, G. Assefa et al., "On-farm performance testing of tropically adaptable chicken strains under small holder management in three countries of sub-Saharan Africa," in Proceedings of the Seventh all Africa Conference on Animal Agriculture, Ethiopian Institute of Agricultural Research, International Livestock Research Institute (ILRI), vol. 9, pp. 293-299, Accra, Ghana, July 2019.

[15] FAO (Food and Agriculture Organization), "Poultry in the 21st century: avian influenza and beyond," in International Poultry Conference BangkokFAO, Rome, Italy, 2007.

[16] D. Temple and X. Manteca, "Animal welfare in extensive production systems is still an area of concern," Frontiers in Sustainable Food Systems, vol. 4, Article ID 545902, 2020.

[17] H. D. Hedman, K. A. Vasco, and L. Zhang, "A review of antimicrobial resistance in poultry farming within low-resource settings," Animals, vol. 10, no. 8, pp. 1264-1339, 2020.

[18] O. Bamidele and T. A. Amole, "Impact of COVID-19 on smallholder poultry farmers in Nigeria," Sustainability, vol. 13, no. 20, Article ID 11475, 2021.

[19] A. Yakubu, O. Bamidele, W. A. Hassan et al., "Farmers' choice of genotypes and trait preferences in tropically adapted chickens in five agro-ecological zones in Nigeria," Tropical Animal Health and Production, vol. 52, no. 1, pp. 95-107, 2020.

[20] N. Elkin, Aminoglycosides, https://www.poultrymed.com/ Aminoglycosides, 2021.

[21] WHO, 2021 AWaRe Classification, WHO, Geneva, Switzerland, 2021.

[22] WHO, Critically Important Antimicrobials for Human Medicine, World Health Organization, Geneva, Switzerland, 6th edition, 2019.

[23] CBN, Central Bank of Nigeria|Exchange Rate, CBN, Abuja, Nigeria, 2021, https://www.cbn.gov.ng/rates/exchrateby currency.asp.
[24] N. V. Hegde, S. Kariyawasam, and C. DebRoy, "Comparison of antimicrobial resistant genes in chicken gut microbiome grown on organic and conventional diet," Veterinary and Animal Science, vol. 1-2, pp. 9-14, 2016.

[25] FAO (Food and Agriculture Organization), Drivers, Dynamics and Epidemiology of Antimicrobial Resistance in Animal Production, FAO, Rome, Italy, 2016.

[26] J. C. Moreki, B. Poroga, R. Dikeme, and D. Seabo, "Ethnoveterinary medicine and health management in poultry in Southern and Western Districts, Botswana," Livestock Research for Rural Development, vol. 22, no. 6, 2010.

[27] O. O. Alabi, F. O. Ajayi, O. Bamidele et al., "Impact assessment of improved chicken genetics on livelihoods and food security of smallholder poultry farmers in Nigeria," Livestock Research for Rural Development, vol. 32, no. 5, p. 77, 2020.

[28] K. Gabanakgosi, J. C. Moreki, S. J. Nsoso, and C. Tsopito, "Ethnoveterinary medicine usage in family chickens in the selected four villages of Botswana," Animal Science, vol. 2, no. 12, pp. 586-594, 2012.

[29] FAO (Food and Agriculture Organization), Small Scale Poultry Production: Technical Guide, FAO, Rome, Italy, 2004.

[30] E. F. Guèye, "Gender issues in family poultry production systems in low-income food-deficit countries," American Journal of Alternative Agriculture, vol. 18, no. 4, pp. 185-195, 2003.

[31] E. F. Guéye, "The role of networks in information dissemination to family poultry farmers," World's Poultry Science Journal, vol. 65, no. 1, pp. 115-124, 2009.

[32] FAO, The Role of Women in AgricultureFAO, Rome, Italy, 2011, https://www.fao.org/3/am307e/am307e00.pdf.

[33] T. Alemayehu, J. Bruno, J. Poole et al., Household Baseline Data in Nigeria: Monitoring Delivery of Chicken Genetic GainsInternational Livestock Research Institute, Nairobi, Kenya, 2018, https://hdl.handle.net/10568/97038.

[34] J. Xu, R. Sangthong, E. Mcneil, R. Tang, and V. Chongsuvivatwong, "Antibiotic use in chicken farms in northwestern China," Antimicrobial Resistance and Infection Control, vol. 6, pp. 1-9, 2020.

[35] L. Gasperini and C. Maguire, "Targeting the rural poor: the role of education and training," Sustainable Development Dimensions, FAO, Rome, Italy, pp. 3-7, 2002.

[36] C. Ducrot, A. Hobeika, C. Lienhardt et al., "Antimicrobial resistance in Africa-how to relieve the burden on family farmers," Emerging Infectious Diseases, vol. 27, no. 10, pp. 2515-2520, 2021.

[37] J. M. Chah, E. M. Igbokwe, and K. Chah, "Ethno-veterinary medicine used in small ruminant health in the Eastern Guinea Savanna, Nigeria," Livestock Research for Rural Development, vol. 21, no. 12, 2009.

[38] Z. Iqbal, A. Jabbar, M. S. Akhtar, G. Muhammad, and M. Lateef, "Possible role of ethnoveterinary medicine in poverty reduction in Pakistan: use of botanical anthelmintics as an example," Journal of Agriculture and Social Sciences, vol. 1, pp. 187-195, 2005.

[39] L. Coyne, R. Arief, C. Benigno et al., "Characterizing antimicrobial use in the livestock sector in three south east Asian countries (Indonesia, Thailand, and Vietnam)," Antibiotics, vol. 8, no. 1, p. 33, 2019.

[40] M. M. Evans, N. N. Samuel, and C. M. Samuel, "Production of indigenous poultry among smallholder farmers in Tigania West Meru County, Kenya," African Journal of Agricultural Research, vol. 17, no. 5, pp. 705-713, 2021.

[41] M. M. Sirdar, J. Picard, S. Bisschop, and B. Gummow, "A questionnaire survey of poultry layer farmers in Khartoum 
State, Sudan, to study their antimicrobial awareness and usage patterns," Onderstepoort Journal of Veterinary Research, vol. 79, no. 1, pp. E1-E8, 2012.

[42] A. Joshua, A. Moses, and A. Ezekiel Olugbenga, "A survey of antimicrobial agents usage in poultry farms and antibiotic resistance in Escherichia coli and staphylococci isolates from the poultry in Ile-Ife, Nigeria," Journal of Infectious Diseases and Epidemiology, vol. 4, no. 1, pp. 4-11, 2018.

[43] A. A. Masud, E. K. Rousham, M. A. Islam et al., "Drivers of antibiotic use in poultry production in Bangladesh: dependencies and dynamics of a patron-client relationship," Frontiers in Veterinary Science, vol. 7, pp. 1-9, 2020.

[44] S. Kiambi, R. Mwanza, A. Sirma et al., "Understanding antimicrobial use contexts in the poultry sector: challenges for small-scale layer farms in Kenya," Antibiotics, vol. 10, no. 2, pp. 106-116, 2021.

[45] J. Ferdous, S. Sachi, Z. Al Noman, S. M. A. K. Hussani, Y. A. Sarker, and M. H. Sikder, "Assessing farmers' perspective on antibiotic usage and management practices in small-scale layer farms of Mymensingh district, Bangladesh," Veterinary World, vol. 12, no. 9, pp. 1441-1447, 2019.

[46] B. Oluwasile, M. Agbaje, O. Ojo, and M. Dipeolu, "Antibiotic usage pattern in selected poultry farms in Ogun state," Sokoto Journal of Veterinary Sciences, vol. 12, no. 1, p. 45, 2014.

[47] R. E. Guetiya Wadoum, N. F. Zambou, F. F. Anyangwe et al., "Abusive use of antibiotics in poultry farming in Cameroon and the public health implications," British Poultry Science, vol. 57, no. 4, pp. 483-493, 2016.

[48] C. Manyi-Loh, S. Mamphweli, E. Meyer, and A. Okoh, "Antibiotic use in agriculture and its consequential resistance in environmental sources: potential public health implications," Molecules, vol. 23, no. 4, 2018.

[49] S. Johnson, K. Bugyei, P. Nortey, and W. Tasiame, "Antimicrobial drug usage and poultry production: case study in Ghana," Animal Production Science, vol. 59, no. 1, pp. 177-182, 2019.

[50] A. S. Ahmed and A. L. Mustapha, "Consumer preferences and willingness to pay for chicken meat traits: a discrete choice experiment approach," Journal of Agricultural Economics, Environment and Social Sciences, vol. 6, no. 1, pp. 131-139, 2020.

[51] F. O. Ajayi, "Nigerian indigenous chicken: a valuable genetic resource for meat and egg production," Asian Journal of Poultry Science, vol. 4, no. 4, pp. 164-172, 2010. 